\title{
TV/Series
}

$2 \mid 2012$

Les séries télévisées dans le monde : Échanges, déplacements et transpositions

\section{La série catalane Ventdelplà, entre réalisme et utopie}

\section{Jennifer Houdiard}

\section{(2) OpenEdition \\ Journals}

Édition électronique

URL : http://journals.openedition.org/tvseries/1384

DOI : 10.4000/tvseries.1384

ISSN : 2266-0909

Éditeur

GRIC - Groupe de recherche Identités et Cultures

Référence électronique

Jennifer Houdiard, "La série catalane Ventdelplà, entre réalisme et utopie », TV/Series [En ligne],

2 | 2012, mis en ligne le 01 novembre 2012, consulté le 19 avril 2019. URL : http://

journals.openedition.org/tvseries/1384; DOI : 10.4000/tvseries.1384

\section{(c) (i) $\odot$}

TV/Series est mis à disposition selon les termes de la licence Creative Commons Attribution - Pas d'Utilisation Commerciale - Pas de Modification 4.0 International. 


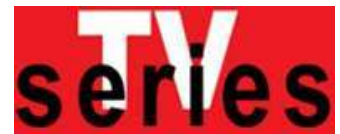

La série catalane Ventdelplà, entre réalisme et utopie

Jennifer HOUDIARD

La série Ventdelplà ( $\mathrm{TV}_{3}$, 2005-2010) porte le nom du village imaginaire dans lequel s'ancre l'essentiel de l'action: Teresa, la protagoniste, fuit Barcelone et son mari qui la maltraite, pour se réfugier dans la demeure de ses ancêtres, au cœur de la campagne gironaise. Les premiers épisodes de la série donnent à voir une nette bipolarisation de l'espace : Teresa laisse derrière elle la grande cité, bruyante et bondée, un époux violent, des amis superficiels, et s'intègre peu à peu à la population de Ventdelplà, entre paysans, commères et notables locaux. Mais l'image du locus amoenus dessinée par les premiers plans sur Ventdelplà se révèle rapidement être un leurre : le petit village paisible n'est pas épargné par les fléaux de la société contemporaine; en revanche, le cadre rural permet d'explorer d'autres problématiques, comme la préservation de l'environnement ou les difficultés rencontrées par les agriculteurs d'aujourd'hui.

Ventdelplà s'inscrit dans la continuité de la production fictionnelle antérieure de $\mathrm{TV}_{3}$, marquée par la recherche du réalisme et de l'authenticité ; elle montre un autre visage de la Catalogne d'aujourd'hui, visage que ses concepteurs veulent aussi « vrai » que celui de la Barcelone qui servait de décor à Poblenou ou à El cor de la ciutat, mais qui se révèle être tout aussi peu vraisemblable du point de vue politique. En effet, Ventdelplà, à l'instar de la majorité des séries catalanes diffusées jusqu'à ce jour, montre une Catalogne à l'identité affirmée et jamais remise en question, uniformément catalanophone, et dont l'existence comme nation semble aller de soi. De plus, si la série s’inscrit explicitement dans un espace identifiable comme réel, le fait que la majeure partie de l'action se déroule dans un village imaginaire mérite que l'on s'interroge: Ventdelplà ne serait-il pas une sorte d'Utopia linguistique, culturelle et politique?

$\mathrm{L}$ a chaîne de télévision TV3 a vu le jour fin 1983, durant la Transition démocratique; sa création s'inscrit dans un contexte de réaffirmation d'une identité nationale catalane mise à mal durant le franquisme ${ }^{1}$. L'emploi presque exclusif de la langue catalane, ainsi que l'ancrage du contenu des programmes dans l'espace catalan, font de TV3, selon une spécialiste, un « outil de normalisation linguistique, de normalisation culturelle et de cohésion nationale ${ }^{2} »$. Après l'information, la fiction est très vite apparue sur la grille des programmes de la chaîne, d'abord en format court, puis sous la forme de productions de plus grande envergure dans les années 1990. L'action des premières séries était majoritairement ancrée à

\footnotetext{
${ }^{1}$ Le rôle de premier plan de la télévision dans la reconstruction du pays a été évoqué par Joan Granados, directeur de la Corporation Catalane de Radio et Télévision, en 1993 : "Quand Televisió de Catalunya est née, il y a maintenant dix ans, elle n'a pas seulement été conçue comme un projet audiovisuel, mais avec l'idée d'en faire un outil fondamental pour le redressement de notre pays. Dans la situation de détérioration linguistique et culturelle qui était la nôtre, nous étions conscients du fait que seul un média de masse, comme la télévision, intégralement en catalan, [...] pourrait contribuer à réduire la grande distance qui nous séparait de la normalité », in TV3. 10 anys, dir. Teresa Guitart, Enric Bajuelo, Barcelone, Columna, 1993, p. 9. Je traduis.

${ }^{2}$ Imma Tubella, Televisió $i$ identitat, thèse dirigée par Joan Becat soutenue à l'université de Perpignan, 1999, p. 278. Je traduis.
} 
Barcelone 3 , à quelques exceptions près ; mais très vite, la volonté des instances dirigeantes de $\mathrm{TV}_{3}$ de proposer à tous les Catalans des fictions proches de leur quotidien a poussé les scénaristes et réalisateurs à explorer de nouveaux espaces, comme les villes moyennes ou les zones rurales. L'un des objectifs de la création de fictions catalanes était de proposer une alternative aux soap operas anglo-saxons et aux culebrones ${ }^{4}$ latino-américains, qui connaissaient un vif succès en Espagne dans les années 1980 et $1990^{5}$.

Ventdelplà ( $\mathrm{TV}_{3}, 2005$-2010) est la première série catalane émise en prime time, avec deux épisodes de cinquante-deux minutes par semaine. Elle a été diffusée de février 2005 à octobre 2010, et prolongée à la demande des téléspectateurs ${ }^{6}$. Selon ses concepteurs, la principale raison du grand succès de Ventdelplà est son réalisme7; la série montrerait le "vrai visage » de la Catalogne et de ses habitants, offrant ainsi un support d'identification à tous les Catalans, jeunes ou âgés, citadins ou villageois. Si Ventdelplà peut être considérée comme une série réaliste par comparaison avec d'autres productions, notamment étrangères, elle semble toutefois construire une image utopique de la Catalogne du point de vue politique, culturel et linguistique. Je tenterai de le démontrer en m'appuyant sur l'analyse des premiers épisodes de la série.

Le début de l'épisode pilote ${ }^{8}$ de Ventdelplà plonge les spectateurs dans un univers fictionnel familier, à plus d'un titre : l'action démarre à Barcelone, où vit Teresa, la protagoniste, avec son mari, Damià, et leurs deux enfants, Isona et Biel (voir figure 1). L'appartement bourgeois et la famille qui l'habite semblent sortir tout droit d'un soap opera ou d'un culebrón. L'organisation par le mari de Teresa d'une fête surprise pour célébrer l'anniversaire de leur union, tout comme le splendide bracelet en or qu'il lui offre, nous immerge dans les clichés du feuilleton sentimental. Quant à Alícia (voir figure 2), l'amie du couple, elle est à elle seule un archétype : cette femme aussi sophistiquée que frivole n'a d'autre occupation dans la vie que de dépenser l'argent qu'elle a obtenu grâce à son divorce, et vit par

3 On peut par exemple penser aux séries Poblenou $\left(\mathrm{TV}_{3}, 1994\right)$ ou El cor de la ciutat (TV3, 2000-2009).

4 On appelle familièrement culebrones (littéralement, «grandes couleuvres ») certaines séries latino-américaines, en raison de leur longueur et de leur sinuosité narrative.

5 Voir Joana Gallego, «Els serials catalans : un nou producte amb denominació d'origen », Anàlisi, $\mathrm{n}^{\circ} 23,1999$, p. 18 [p. 17-24].

${ }^{6}$ La fin de la série a été annoncée officiellement à la fin de la troisième saison, en 2007 , mais, suite à une importante mobilisation du public, quatre autres saisons ont vu le jour.

7 Voir L'enigma Ventdelplà, reportage diffusé sur TV3 le 27 avril 2008, consultable à l'adresse suivante : http://www.tv3.cat/videos/404769, lien consulté le 29 août 2011.

8 Épisode 1, diffusé le 15 février 2005 :

http://www.ventdelpla.cat/pprogrames/ventdelpla/vdpSeccio.jsp?seccio=temporada\&idint $=174942769 \&$ num $=1$. Consulté le 29 août 2011. Les épisodes n'étant pas numérotés par saison, seul le numéro de l'épisode sera précisé. 
procuration la relation qui unit Teresa et Damià. Sa tendance à chanter en permanence les louanges du mari de son amie, qui représente pour elle l'homme parfait, la construit comme une briseuse de couple potentielle : elle incarne la femme fatale, la séductrice, ingrédient indispensable à toute fiction sentimentale qui se respecte. Ainsi, le monde que nous découvrons de prime abord n'est pas sans évoquer un type de fiction dont $\mathrm{TV}_{3}$ a toujours souhaité se démarquer. Dans l'univers feutré d'un quartier bourgeois de Barcelone, Teresa figure une femme au foyer comblée, épouse d'un homme séduisant et prospère, et mère de deux beaux enfants ; la seule ombre au tableau en puissance semble être la proximité de l'amie-rivale : rien de très original, en somme. Ce panorama semble aisément transposable dans une banlieue chic de Californie ou dans les beaux quartiers d'une ville latinoaméricaine.

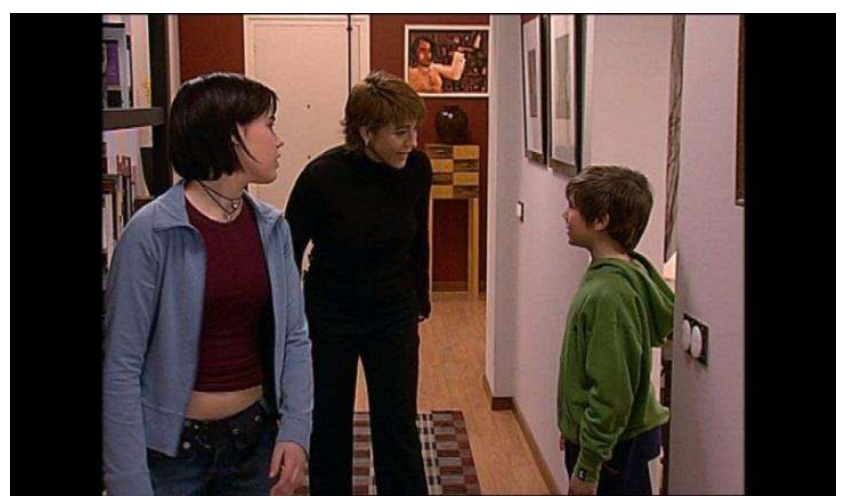

Fig. 1 : Teresa et ses enfants dans leur appartement barcelonais (épisode 1)

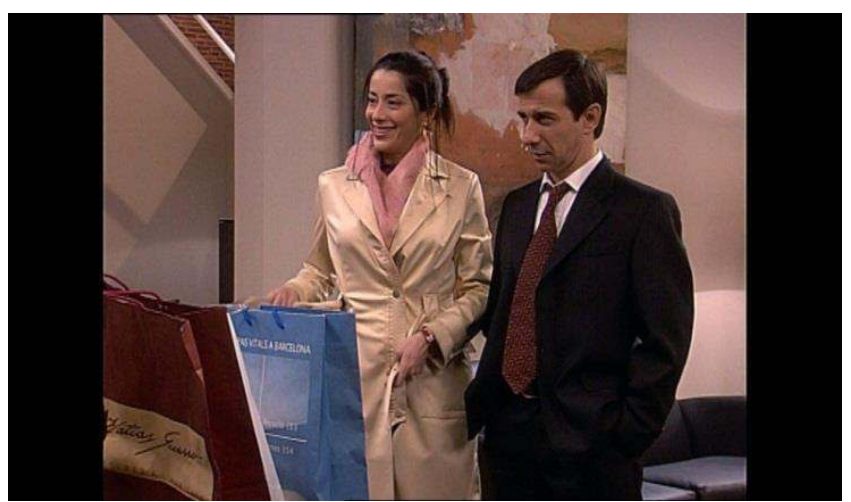

Fig. 2 : Alícia et Damià (épisode 1) 
En réalité, tout se passe comme si le cliché était recréé pour mieux être déconstruit. C'est d'abord la représentation de l'espace extérieur qui vient nuancer quelque peu les premières impressions : lorsque la caméra quitte l'impeccable appartement de Teresa et de Damià, les téléspectateurs sont plongés sans ménagements dans l'ambiance oppressante de la grande ville. Les plans très rapprochés et flous sur la circulation et les rues bondées se succèdent à un rythme rapide, associant d'emblée le cadre urbain à l'inconfort et au stress ; les bruits de klaxons et de moteurs achèvent de construire un univers hostile. Rapidement, Barcelone est caractérisée négativement, et le monde dans lequel évolue la protagoniste l'est également.

En effet, il apparaît assez vite que Teresa est une «femme au foyer désespérée », au sens littéral, et son cadre de vie idyllique, une cage dorée, à l'image du bracelet que lui offre Damià pour commémorer leurs dix-huit ans de relation, un «bracelet esclave » en or qui n'aura jamais aussi bien porté son nom. L'on apprend rapidement que si Teresa a cessé d'exercer la médecine, c'est en raison de l'insistance de Damià, qui désirait que sa femme reste à la maison. D'ailleurs, il n'accepte pas qu'elle travaille ponctuellement comme infirmière. Lorsqu'elle lui explique qu'elle a besoin d'un peu d’indépendance, il lui réplique brutalement : " Il faut l'admettre, tu n'es pas indépendante, tu dépends de moi » (épisode 1). Peu à peu, l'envers du décor de la vie de Teresa est dévoilé : l'épouse de l'avocat brillant, la mère de famille comblée est en réalité victime de violences conjugales. Si elle semble à l'abri du monde extérieur, du bruit et de l'agitation des rues saturées de Barcelone, c'est parce qu'elle en est coupée : les seules personnes qu'elle fréquente sont les amis de Damià ; elle est isolée. La solitude de la protagoniste à Barcelone apparaît au spectateur dans toute sa cruauté lorsqu'elle décide d'expliquer à Alícia que son mari la maltraite : cette dernière reproche d'ordinaire à Teresa d'être trop jalouse de sa vie privée, et se réjouit lorsque son amie lui annonce un déjeuner au restaurant et des confidences. Cependant, le sourire d'Alícia se fige lorsque le thème des mauvais traitements est abordé. Elle finit par couper sèchement la parole à Teresa en lui disant : "Ton mari est un homme merveilleux, et il est très amoureux de toi » (épisode 1). Alícia refuse que l'histoire d'amour idéale qu'elle a sous les yeux ne soit qu'une fiction inventée de toutes pièces par Damià. Pour échapper à la vérité insupportable qui lui est dépeinte, Alícia invente une autre fiction : Teresa aurait un amant et chercherait une excuse pour pouvoir quitter son époux légitime. Ce personnage de feuilleton à l'eau de rose semble essayer de maintenir autour d'elle le décor qui lui est assorti ; cependant, elle contribue à le faire voler en éclats, en révélant à quel point la protagoniste est seule à Barcelone.

De fait, Teresa ne peut trouver de soutien qu'en la personne de son oncle Gustau, qui vit à Ventdelplà (voir figure 3), le petit village dont la protagoniste est originaire. C'est à travers ce personnage 
qu'apparaît un «ailleurs » et que commence à se dessiner la bipolarisation qui caractérise l'espace fictionnel de la série : lors des conversations téléphoniques qu'il partage avec sa nièce dans le premier épisode, Gustau est filmé dans sa vieille maison rurale, un décor chaleureux, chargé d'objets personnels et rempli de livres, qui contraste avec l'appartement froid et impersonnel de Teresa et Damià à Barcelone. À mesure que les spectateurs découvrent la triste existence de la protagoniste féminine dans une ville qui lui est devenue étrangère et où elle est comme seule au monde, le montage en parallèle de certaines séquences montre un autre univers : celui d'un intellectuel philanthrope menant une vie paisible dans un village qui se présente de prime abord, par contraste avec Barcelone, comme un véritable petit paradis. Quand il n'est pas occupé à écrire, Gustau (voir figure 4) aide ses deux jeunes protégés à préparer leurs examens, corrige leurs copies lors de ses promenades dans les rues calmes de Ventdelplà, ou rejoint ses amis pour une partie de cartes au bar Tramuntana.

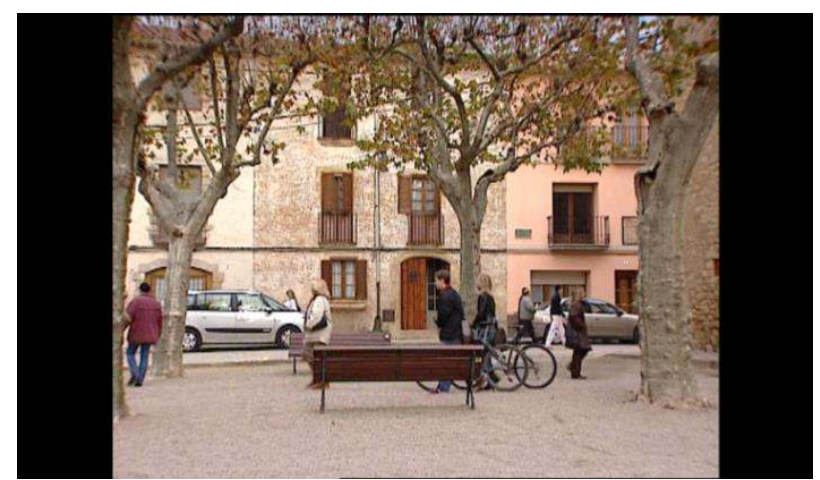

Fig. 3 : La place de la mairie de Ventdelplà (épisode 1)

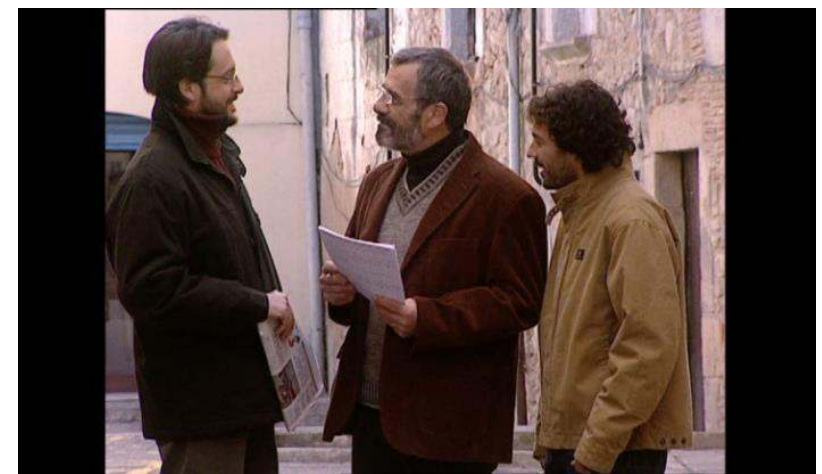

Fig. 4 : L’oncle Gustau (au centre) dans une rue de Ventdelplà (épisode 1) 
L'harmonie et la convivialité semblent régner dans le village ; c'est en tout cas ce que suggère le générique de la série. Les premières images du générique, qui montrent des rues saturées de voitures et de piétons, rappellent les plans extérieurs de Barcelone ponctuant les premiers épisodes, mais laissent rapidement la place à des plans larges sur de grands espaces, où le vert domine, puis à diverses vues du petit village catalan de Breda, où sont tournés les extérieurs de la série. La présentation des rôles principaux est agrémentée de diverses images qui évoquent le quotidien à Ventdelplà : la terrasse d'un bar, des vaches dans une étable, une partie de cartes. Le générique se termine sur une vue aérienne bucolique du village (voir figure 5), dont on devine qu'il deviendra rapidement le décor de la vie de la protagoniste ; c'est tout du moins ce que laissent penser les paroles de la chanson interprétée par Gerard Quintana9 : Ventdelplà sera « l'ailleurs » où Teresa pourra « tout recommencer » et reprendre en main les rênes de sa vie.

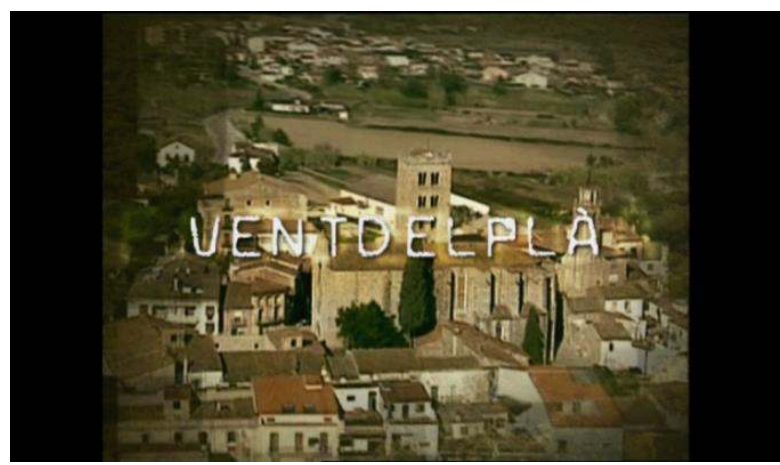

Fig. 5 : Image finale du générique de la série

C'est sur cette idée de nouveau départ que se clôt le premier épisode : après avoir été giflée par son mari sous les yeux de son fils, la protagoniste part se réfugier chez son oncle Gustau. Un peu avant d'arriver à destination, elle arrête la voiture au bord de la route, et réveille ses enfants pour leur montrer le village, niché au creux d'une vallée, prononçant avec émotion son nom : "Voici Ventdelplà » (épisode 1). L’image de la mère et de ses enfants contemplant le décor de leur nouvelle vie à venir est accompagnée par la voix de Teresa, en off, qui annonce: "Maintenant, les choses seront différentes » (épisode 1).

9 Le texte est le suivant: «Découvre-toi / Et tu sentiras que le chemin est immense / Trouve un ailleurs pour tout recommencer / Construis ta vie / Au rythme des battements de ton cœur / Ouvre les yeux sur le monde / C'est toi qui décidera », extrait de la chanson " Tu descobriràs » (texte de Antoni-Olaf Sabater), 2005. Je traduis. 
Effectivement, c'est dans un nouveau monde qu'atterrissent la protagoniste et ses deux enfants : un petit village au cour d'un paysage verdoyant et paisible, où tout le monde se connaît, où les portes des maisons restent ouvertes lorsque leurs propriétaires s'absentent. Lorsque Teresa, quelques jours après sa fuite, retourne à Barcelone pour récupérer les affaires laissées dans son ancien appartement, le gros plan sur l'impressionnante serrure de la porte blindée souligne le contraste (épisode $5^{10}$ ): la grande ville, lieu de tous les dangers, condamne ses habitants à s'enfermer et, par là même, à s'isoler ; à l'inverse, à Ventdelplà, chacun est protégé par la bienveillance de tous, les habitants se connaissent et se font confiance. D'ailleurs, les téléspectateurs se sentent très rapidement chez eux : le nombre de personnages et de lieux étant limité, ils sont parfaitement connus dès la fin du troisième épisode. Tout est mis en œuvre pour créer une sensation de familiarité; la quasi-totalité des séquences qui se déroulent en intérieur sont introduites par un plan extérieur du lieu, contribuant ainsi à créer une représentation globale du village de Ventdelplà dans l'esprit des téléspectateurs. Cette technique permet aussi de faciliter l'illusion référentielle, car les intérieurs, tournés en studio, sont montrés comme ancrés dans la réalité du village, dans les maisons dont on finit rapidement par reconnaître les façades et par identifier les habitants : la maison de l'oncle Gustau, sur la place de la mairie, celle de David, l'avocat avec qui Teresa se lie d'amitié, ou encore le bar Tramuntana. Le site web dédié à la série (voir figure 6) achève de brouiller les pistes entre fiction et réalité, en proposant au public un plan de Ventdelplà sur lequel sont marqués les principaux espaces dans lesquels se déroule l'action, ainsi qu'une série de photographies du village ${ }^{11}$.

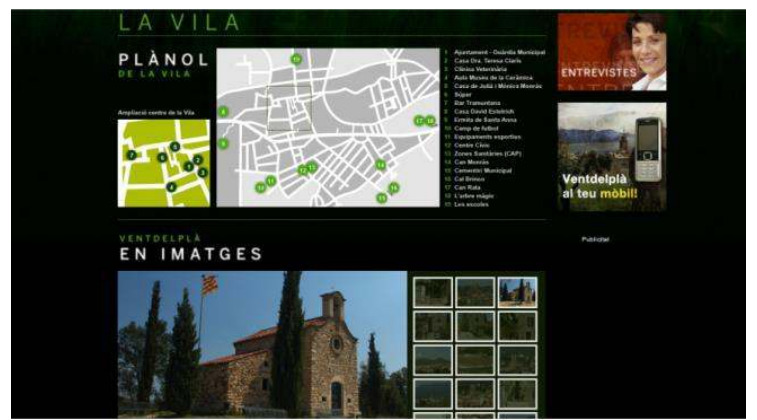

Fig. 6 : Page du site officiel consacré à la série

1o Épisode diffusé le $1^{\text {er }}$ mars 2005 :

http://www.ventdelpla.cat/pprogrames/ventdelpla/vdpSeccio.jsp?seccio=temporada\&idint $=176334172 \&$ num $=1$. Consulté le 29 août 2011.

${ }^{11}$ http://www.ventdelpla.cat/la_vila.html\#/img/la_vila/3.jpg. Consulté le 29 août 2011. 
La volonté de créer un sentiment de proximité chez les téléspectateurs se double d'une recherche de réalisme qui vient assez vite nuancer l'image «idéale» du petit village. La dichotomie Ventdelplà/Barcelone se maintient, mais dépasse rapidement le plan axiologique : il n'y a rapidement plus de «bon » ou de "mauvais » espace, mais un simple déplacement de la protagoniste, et, du même coup, de l'action. L'installation de Teresa dans le village est, certes, un nouveau départ, mais son chemin n'en est pas moins semé d'embûches. Lors de son arrivée, elle apprend le décès brutal de son oncle Gustau (épisode $2^{12}$ ). Peu après, Damià coupe les vivres à sa femme pour se venger d'avoir été quitté ; elle se retrouve sans ressources et contrainte de mettre en vente la maison de son oncle ainsi que sa bibliothèque (épisode $3^{13}$ ). Lors de son arrivée, Teresa était pour les habitants une étrangère, une femme de la grande ville, et ne suscitait guère plus que de la curiosité. Mais lorsque la nouvelle de la vente des biens de Gustau se répand dans le village, la nouvelle venue devient la cible de toutes les médisances et de toutes les attaques : le maire lui-même vient l'accuser de vendre la mémoire de Ventdelplà en vendant les archives de Gustau. Quant aux commères (hommes et femmes) du village, ils organisent une manifestation afin d'humilier Teresa: le jour où l'acheteur vient récupérer les archives, c'est dans un fracas de casseroles et sous les insultes qu'ils stigmatisent la «profiteuse », la «voleuse » (voir figure 7).

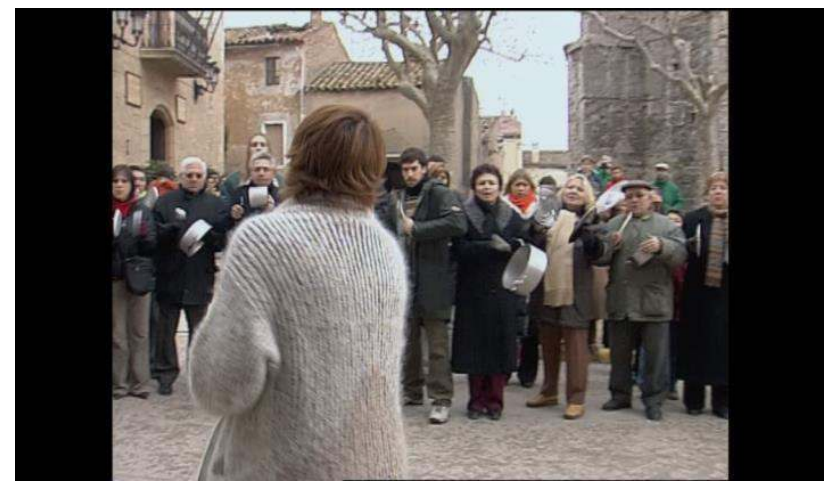

Fig. 7 : Manifestation des villageois contre Teresa (épisode 8)

12 Épisode diffusé le 16 février 2005 :

http://www.ventdelpla.cat/pprogrames/ventdelpla/vdpSeccio.jsp?seccio=temporada\&idint $=175011760 \&$ num $=1$. Consulté le 29 août 2011.

${ }^{13}$ Épisode diffusé le 22 février 2005 :

http://www.ventdelpla.cat/pprogrames/ventdelpla/vdpSeccio.jsp?seccio=temporada\&idint $=175629068 \&$ num=1 . Consulté le 29 août 2011. 
Cette longue séquence (épisode $8^{14}$ ), assez violente, achève de faire de Teresa un personnage d'exclue, plus seule que jamais. Ainsi, en quelques épisodes, le locus amoenus est devenu un lieu hostile : la protagoniste a fui l'enfer, mais devra lutter pour se construire une nouvelle vie à Ventdelplà.

L'attitude des habitants du village face à Teresa permet toutefois une autre lecture. En effet, "l'étrangère », victime du rejet, est également la personnification d'une menace pour le patrimoine du village : en tant que Barcelonaise, elle représente ceux qui colonisent peu à peu les campagnes pour y établir leur résidence secondaire, ou simplement pour s'enrichir en spéculant. Lorsque Teresa reçoit la visite d'un potentiel acheteur pour la maison (épisode 3), celui-ci ne fait pas mystère de ses projets : il n'est intéressé que par le terrain, sur lequel il souhaite construire un immeuble de standing. S'il accepte de conserver la façade, c'est uniquement parce que cela permettra d'augmenter le prix des appartements : l'homme est un investisseur, et n'a que faire du patrimoine architectural de Ventdelplà. Ce personnage sans scrupules éclaire quelque peu l'attitude des habitants du village : l'attaque contre Teresa peut être interprétée comme un acte défensif, par lequel les villageois refusent que leur patrimoine, leur mémoire, puissent être vendus au plus offrant. C'est un problème bien réel qui est évoqué ici : la spéculation immobilière est une véritable menace pour les villages tels que Ventdelplà. D'ailleurs, tout au long des sept saisons de la série, les effets néfastes des invasions citadines sur les petits villages catalans sont rappelés régulièrement: dans la sixième, par exemple, un agriculteur se voit interdire de fertiliser ses champs durant les weekends, pour ne pas troubler l'air pur que son voisin barcelonais vient respirer à la campagne (épisode $313^{15}$ ).

Même si le temps semble s'être un peu arrêté à Ventdelplà, même si la convivialité et la simplicité y font encore un peu de résistance, le village n'est pas pour autant à l'abri des fléaux de la société contemporaine. L'Amsterdam, que certains habitants surnomment pudiquement «le bar de la route », est l'endroit où l'agriculteur Jaume vient oublier sa solitude et chercher un peu d'affection tarifée : sa femme l'a quitté, ses deux fils aînés sont partis travailler à l'étranger. Le petit dernier a choisi de rester au village et de se consacrer à la terre, mais il a rejoint une coopérative d'agriculture biologique, laissant Jaume totalement seul et accablé de travail. Peu à peu, la caméra glisse de la triste vie du fermier à une autre détresse :

14 Épisode diffusé le 9 mars 2005 :

http://www.ventdelpla.cat/pprogrames/ventdelpla/vdpSeccio.jsp?seccio=temporada\&idint $=177122605 \&$ num $=1$. Consulté le 29 août 2011.

15 Épisode diffusé le 21 septembre 2009 :

http://www.ventdelpla.cat/pprogrames/ventdelpla/vdpSeccio.jsp?seccio=temporada\&idint $=1496009 \&$ num $=6$. Consulté le 29 août 2011. 
celle des prostituées de l'Amsterdam, qui sont majoritairement des étrangères séquestrées par le propriétaire du bar. Lorsque l'une d'entre elles tente de fuir et se réfugie au bar de Ventdelplà (épisode $9^{16}$ ), deux univers se télescopent ; le monde protégé des habitants du village entre en contact avec une violence et une misère extrêmes, subies par une femme qui n'a d'autre choix que de vendre son corps en espérant, une fois sa « dette » payée, récupérer son passeport et retrouver sa liberté. À travers le personnage de Daisy, les spectateurs voient sans fard le « monde de la nuit » des routes catalanes, trop souvent considéré avec bienveillance ou indifférence par l'opinion publique.

Ventdelplà peut donc être considérée comme une série réaliste, en prise avec les problèmes de la société catalane contemporaine ; selon les déclarations du réalisateur, la volonté de vraisemblance et d'actualité est au cœur des préoccupations des instances dirigeantes de la chaîne : " La première condition posée par TV3 est qu'ils ne veulent pas de ruralisme bon marché [...]. Ils veulent un village actuel, moderne, normal ${ }^{17}$ ». Les habitants de la Catalogne rurale sont censés pouvoir s'identifier aux personnages de la série, ou tout du moins reconnaître l'univers construit par la fiction comme proche du leur.

Toutefois, le réalisme a ses limites lorsque l'on s'aventure sur le territoire de l'identité catalane et de sa représentation dans la fiction. Je me limiterai ici à la question de la langue, même si d'autres aspects mériteraient d'être commentés. La série Ventdelplà donne à voir aux téléspectateurs un univers uniformément catalanophone, dans lequel la langue castillane ${ }^{18}$ n'apparaît que de manière extrêmement sporadique. Bien sûr, la diversité de la population vivant en Catalogne est montrée : certains habitants de Ventdelplà sont originaires d'autres régions d'Espagne; le village accueille également quelques immigrés venus d'Amérique hispanique, des pays de l'Est ou d'Afrique. Cependant, le traitement de ces personnages mérite commentaire. J'évoquerai essentiellement celui de Rafa González (voir figure 8), l'un des jeunes protégés de l'oncle de Teresa. Ce jeune homme est issu d'une des familles les plus modestes du village, originaire d'Andalousie : le père et le plus jeune fils sont ouvriers dans une usine, la mère reste au foyer et arrondit les fins de mois en effectuant des travaux de couture, tandis

\footnotetext{
${ }^{16}$ Épisode diffusé le 14 mars 2005 :

http://www.ventdelpla.cat/pprogrames/ventdelpla/vdpSeccio.jsp?seccio=temporada\&idint $=177898624 \&$ num $=1$. Consulté le 29 août 2011.

${ }_{17}$ Propos de Lluís Maria Güell, cités dans Identitat cultural i societat a les séries de ficció catalanes: dels discursos a la recepció, Enric Castelló, Bernat López, Estudis de Comunicació de la URV, Tarragone, Universitat Rovira i Virgili, 2007, p. 28. Je traduis.

18 Il s'agit de la langue de l'État espagnol. En Catalogne, l'une des 17 communautés autonomes qui forment l'Espagne, le catalan est langue co-officielle. L'Espagne compte plusieurs autres communautés bilingues, comme le Pays Basque, la Galice ou le Pays Valencien.
} 
que Rafa, le fils aîné, finance ses études en travaillant comme serveur au bar Tramuntana.

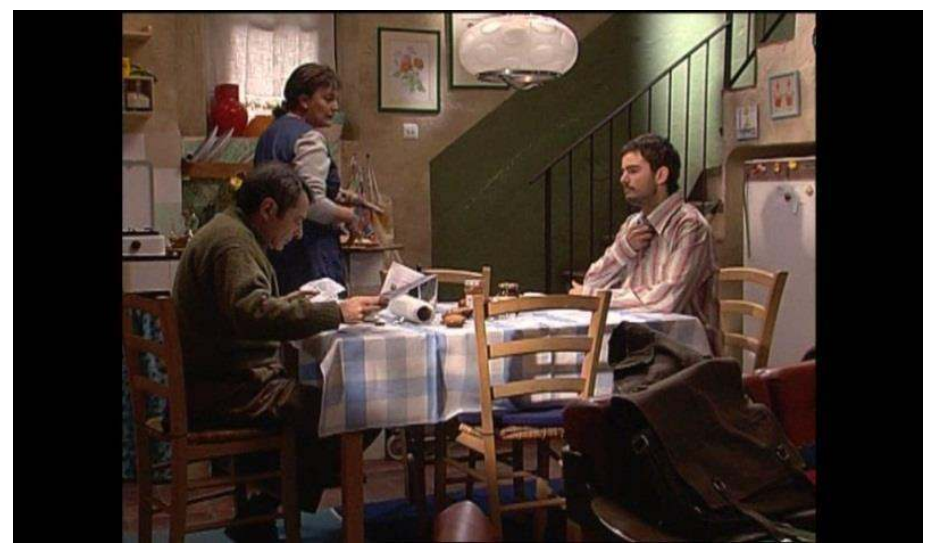

Fig. 8 : Rafa González et ses parents (épisode 3)

Les séquences dans lesquelles apparaît cette famille sont intéressantes du point de vue linguistique. Les parents parlent entre eux en castillan, mais n'utilisent jamais cette langue pour s'adresser à leurs enfants, et pas davantage lorsqu'ils se trouvent à l'extérieur : le castillan est réservé à l'intimité du couple. Quant aux deux enfants, ils s'expriment uniquement en catalan, et les premiers épisodes de la série évoquent copieusement l'attachement du jeune Rafa à la terre d'accueil de ses parents : il a choisi d'étudier la langue et la littérature catalanes à l'université, et écoute de la sardane, une musique traditionnelle. Ce personnage figure un modèle d'intégration réussie, et pourrait être lu comme l'incarnation d'une sorte de "rêve catalan ». Son ascension sociale, mise en parallèle avec son intégration culturelle et linguistique, peut faire l'objet d'une lecture politique: Ventdelplà promeut un modèle de société où l'assimilation favorise la réussite et s'impose comme une évidence, sans jamais poser le moindre problème. L'ouvrier agricole équatorien de Jaume, Alejandro (épisode $15^{19}$ ), après un séjour de quelques mois, comprend parfaitement le catalan, même s'il ne le parle pas -heureusement pour lui, car c'est dans cette langue que tout le monde s'adresse à lui. Quant à la femme d'Alejandro, récemment arrivée à Ventdelplà, c'est tout naturellement que la propriétaire du supermarché s'adresse à elle en catalan, avant de dire à une autre cliente, comme si elle était face à un véritable problème :

19 Épisode diffusé le 4 avril 2005 :

http://www.ventdelpla.cat/pprogrames/ventdelpla/vdpSeccio.jsp?seccio=temporada\&idint $=180057352 \&$ num=1. Consulté le 29 août 2011. 
"Ah. Je crois qu'elle ne nous comprend pas » (épisode 15). C'est seulement lorsque l'impossibilité de communiquer en catalan est avérée que le passage au castillan est envisagé, en dernier recours : la langue de l'Etat espagnol apparaît comme une véritable langue étrangère, pratiquement exclue de l'espace public. Le nouvel arrivant est invité à s'adapter rapidement à son nouvel environnement linguistique, et à réserver sa langue maternelle à la sphère privée. Selon la consultante linguistique d'une autre série de $\mathrm{TV}_{3}$, le traitement de la langue dans les fictions véhicule également une sorte de "code de conduite » à destination des catalanophones : " La fonction de la série avec les personnages d'immigrés est également de 'donner des pistes' aux gens sur la manière dont il faut agir linguistiquement face à des communautés qui ne parlent pas catalan [...]. Dans ce sens, on peut dire que la série de fiction intègre certains types de stratégies pour promouvoir le catalan ${ }^{20} »$. Ces remarques peuvent s'appliquer également à Ventdelplà.

De manière générale, lorsqu'une figure d'étranger accède au statut de personnage récurrent, elle tend à se « catalaniser » au fil des épisodes. C'est le cas de Svetlana, la prostituée russe qui se lie d'amitié avec l'agriculteur Jaume et finira par l'épouser : lors de ses premières apparitions, elle parle un mélange de castillan et de catalan, le tout avec un fort accent slave (épisode $9^{21}$ ). Deux saisons plus tard, elle s'exprime dans un catalan parfait, et pour cause : Georgina Cardona, l'actrice qui l'incarne, est catalane. Il semble légitime de s'interroger sur le choix de la production, qui n'aurait sans doute pas eu de difficultés à trouver à Barcelone une actrice russe pour jouer le rôle de Svetlana. C'est d'autant plus troublant que Nicole, une Australienne qui apparaît quelques saisons plus tard, est également interprétée par une Catalane, Ivana Miño, ce qui explique la promptitude avec laquelle le personnage acquiert une maîtrise enviable de la langue, après seulement quelques mois de séjour au sein de la coopérative d'agriculture biologique de Ventdelplà ${ }^{22}$.

L'intégration, notamment sur le plan linguistique, apparaît comme une évidence, et c'est tout naturellement et avec une facilité déconcertante que les nouveaux venus adoptent le catalan, donné à entendre comme la seule langue légitime dans l'espace public de leur terre d'accueil. Cela est à mettre en rapport avec le fait que les seules forces de l'ordre montrées dans la série sont les Mossos d'Esquadra ${ }^{23}$,

${ }^{20}$ Propos de Núria Comas, consultante linguistique de la série El cor de la ciutat (TV3, 2000-2009), recueillis dans Castelló \& López, p. 33. Je traduis.

${ }^{21}$ Épisode diffusé le 14 mars 2005:

http://www.ventdelpla.cat/pprogrames/ventdelpla/vdpSeccio.jsp?seccio=temporada\&idint $=177898624 \&$ num $=1$. Consulté le 29 août 2011.

${ }^{22}$ Le personnage de Nicole apparaît dans la troisième saison de Ventdelplà.

${ }^{23}$ Force de police catalane qui dépend de la Generalitat, le gouvernement autonome. 
et qu'il n'est jamais question de la Guardia Civil ${ }^{24}$; de la même façon, les institutions de l'Etat espagnol ne sont jamais mentionnées, alors que la Generalitat, le gouvernement autonome catalan, est régulièrement nommée. C'est sur cet aspect que l'on peut prendre en défaut le réalisme de Ventdelplà : tout en prétendant montrer la société catalane contemporaine et le monde rural tels qu'ils sont, sans fard ni idéalisation, la série construit un monde politiquement, culturellement et linguistiquement idéal. Ventdelplà est un village déconnecté des problématiques de la nation sans État qu'est la Catalogne (ce qui a d'ailleurs été reproché à la chaîne TV 3 par des téléspectateurs ${ }^{25}$ ) ; ancré dans l'espace catalan, il figure un "ailleurs» non seulement par rapport à la grande ville, décor habituel des séries catalanes, mais aussi vis-à-vis de la réalité et du chemin qu'il reste encore à parcourir pour la construction (ou reconstruction) de la nation et de l'identité catalane. $\mathrm{Si}$, selon le spécialiste Enric Castelló, les fictions télévisées sont un «élément de projection d'une réalité nationale ${ }^{26}$ ", nous pourrions compléter cette affirmation en ajoutant que l'espace symbolique créé par la fiction est, bien souvent, un espace utopique.

\section{Bibliographie}

Busquets Gemma, «Ventdelplà, com Arenys de Munt », El Punt, 25 septembre 2009, p. 38.

CASTELlo Enric, «La construcció nacional a les sèries de ficció : visió sobre una dècada de producció de Televisió de Catalunya », Quaderns del CAC, $\mathrm{n}^{\circ}$ 23-24, 2005-2006, p. 199-209.

CASTELLO Enric et Bernat LOPEZ, Identitat cultural $i$ societat a les séries de ficció catalanes: dels discursos a la recepció, Estudis de Comunicació de la URV, Tarragone, Universitat Rovira i Virgili, 2007.

GALLEGO Joana, «Els serials catalans: un nou producte amb denominació d'origen ", Anàlisi, n²3, 1999, p. 17-24.

${ }^{24}$ Force de police de l'État espagnol, à statut militaire.

25 La journaliste Gemma Busquets évoque la manière dont la série intègre des éléments du réel, et déplore que Ventdelplà ne s'aventure pas sur le terrain politique : « Maintenant [...] que les scénaristes sont si attentifs aux événements qui secouent la Catalogne, pourquoi ne politisent-ils pas davantage la série ? Ils devraient oser faire de Ventdelplà l'un des villages participant à la consultation populaire pour l'indépendance en suivant l'exemple d'Arenys de Munt [village dans lequel fut organisée le premier scrutin consultatif] ", "Ventdelplà, com Arenys de Munt », El Punt, 25 septembre 2009, p. 38. Je traduis.

${ }^{26}$ Enric Castelló, « La construcció nacional a les sèries de ficció : visió sobre una dècada de producció de Televisió de Catalunya », Quaderns del CAC, n²3-24, 2005-2006, p. 201 [p. 199-209]. 
Guitart Teresa et Enric Bajuelo (dir.), TV3. 10 anys, Barcelone, Columna, 1993.

Tubella Imma, Televisió $i$ identitat, thèse dirigée par Joan Becat soutenue à l'université de Perpignan, 1999. 